\title{
IMPLEMENTACIÓN DE LA AGERDA 2030 A TRAVÉS DE UN PROYECTO DE INVESTIGACIÓN-ACCIÓN APLICARDO LA METODOLOGÍA FLIPPED CLASSROOM
}

\section{Implementation of the 2030 Agenda through an Action-Research Project Applying the Flipped Classroom Methodology}

\author{
María Esperanza Calvo Centeno \\ Universidad Europea de Madrid \\ Email: esperanza.calvo@universidadeuropea.es \\ ORCID: https://orcid.org/0000-0002-4562-8796 \\ Julieta Espín Ocampo \\ Universidad Europea de Madrid \\ E-mail: julieta.espin@universidadeuropea.es \\ ORCID: https://orcid.org/0000-0002-7799-6438
}

\author{
María José Peset González \\ Universidad Europea de Madrid \\ E-mail: mariajose.peset@universidadeuropea.es \\ ORCID: https://orcid.org/0000-0003-2575-1364

Moisés Ruiz González
Universidad Europea de Madrid
E-mail: moises.ruiz@universidadeuropea.es
ORCID: https://orcid.org/0000-0003-4809-3771

Dutores

El propósito del proyecto de investigación-acción, en el que se enmarca la actividad objeto del presente estudio, es incorporar en las áreas de conocimiento de los futuros egresados, el mensaje contenido en la Agenda 2030 de Naciones Unidas con el objetivo de que lo interioricen y, así, favorecer que puedan incorporarlo a su actividad profesional o laboral.

La metodología utilizada para llevar a cabo la actividad es flipped classroom o aula invertida, por considerar que es la metodología más adecuada para que el alumno profundice en los problemas a los que se enfrenta el planeta, sea consciente de los mismos y, en consecuencia, adquiera las competencias necesarias que le permitan aportar soluciones.

El equipo investigador está formado por profesores que imparten docencia en tres áreas de conocimiento adscritas a la Facultad de Ciencias Sociales y de la Comunicación de la Universidad Europea de Madrid. El proyecto de investigación es de carácter multidisciplinar, habiéndose aplicado en cinco asignaturas diferentes, que, a su vez, se imparten en cuatro grados distintos.

Los resultados obtenidos a través de la encuesta realizada muestran que el 85,83\% de los estudiantes tienen un alto grado de satisfacción con la actividad a nivel global y valoran muy positivamente las competencias desarrolladas en la actividad.

Proyecto de investigación-acción; Objetivos de Desarrollo Sostenible; flipped classroom; multidisciplinar; competencias.

Key uords Action-research project; Sustainable Development Goals; flipped classroom; multidisciplinary skills. 
This study is framed into research-action project. Its main goal is to incorporate in the areas of knowledge of future graduates the message of the United Nations 2030 Agenda with the aim of assimilate it and, thus, encourage students to incorporate it into their professional or work activity. The methodology used to carry out the activity is flipped classroom. We consider that it is the most appropriate methodology for students to delve into the issues that the planet faces, to be aware of them and, consequently, acquire the necessary skills that allow them to provide solutions.

The research team is made up of professors who teach in three different areas of knowledge attached to the Faculty of Social Sciences and Communication of the European University of Madrid. The Research Project is multidisciplinary in nature, having been applied in five different subjects, which, in turn, are tanght in four different degrees.

The results obtained through the survey carried out show that $85.83 \%$ of the students have a high degree of satisfaction with the activity on a global level and that they highly value the skills developed in the activity.

\section{Introducción}

Actualmente, el mundo se encuentra inmerso en profundos cambios. La población mundial aumenta a un ritmo anual de unos 80 millones de personas y se prevé que para 2050 el $70 \%$ de la población vivirá en las ciudades. De aquí a 2030 la mayoría de la población mundial en situación de pobreza se concentrará en países afectados por conflictos y en el África subsahariana (Comisión Europea, 2016).

La respuesta a estas tendencias se ha consensuado a nivel internacional a través de la Agenda 2030 para el Desarrollo Sostenible, adoptada en septiembre de 2015 por la Asamblea General de las Naciones Unidas, quedando patente que la sostenibilidad es fundamental para aplicar soluciones a largo plazo a retos mundiales complejos.

La Agenda 2030 está conformada por los Objetivos de Desarrollo Sostenible (ODS) (gráfico 1), basados en los derechos humanos universales (Naciones Unidas, 2015).

Gráfico 1. Objetivos de Desarrollo Sostenible

\section{OBJETIVOS SEOSTERNIBLE}

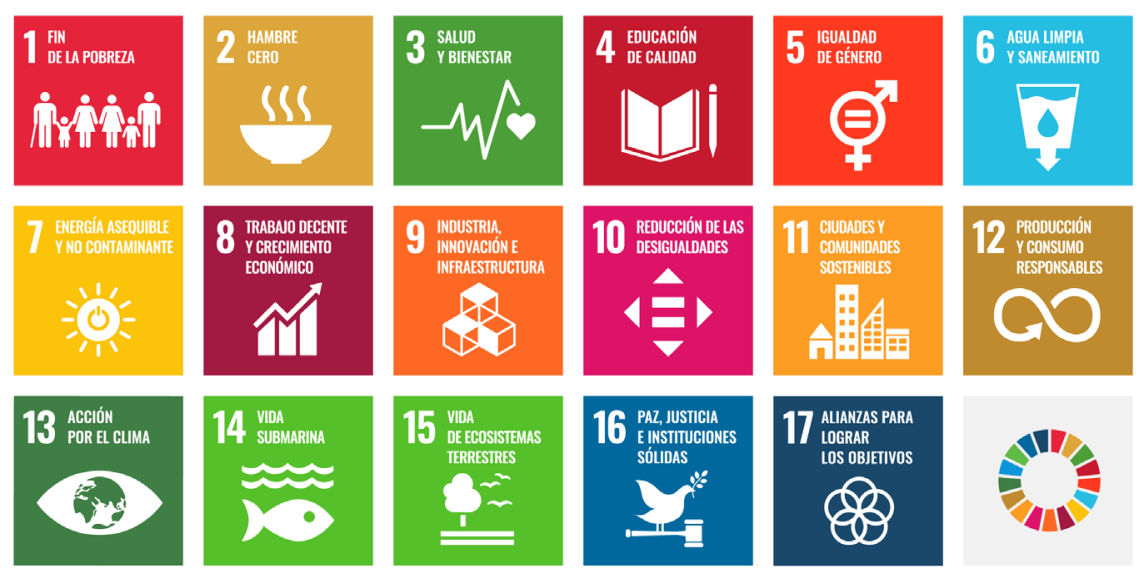

Fuente: Programa de las Naciones Unidas para el Desarrollo (2019) 
Estos objetivos instan a todos los países a adoptar medidas en dos vertientes: promover la prosperidad y proteger al planeta. Los ODS reconocen que para erradicar la pobreza se tienen que implementar estrategias que favorezcan el crecimiento económico y aborden una serie de necesidades sociales, entre las que se encuentra la educación.

El objetivo n. ${ }^{o} 4$ reconoce la importancia de garantizar una educación inclusiva, equitativa y de calidad y promover oportunidades de aprendizaje durante toda la vida para todos. La meta 4.7 del ODS n. ${ }^{\circ} 4$ hace alusión explícita a la necesidad de que todos los alumnos adquieran los conocimientos teóricos y prácticos necesarios para promover el desarrollo sostenible (Naciones Unidas, 2015). Todos deben aprender a entender el complejo mundo en el que viven y ser capaces de colaborar, hablar y actuar por un cambio positivo (UNESCO, 2015).

Entre los objetivos de aprendizaje para docentes recogidos por la UNESCO (2017) para promover el desarrollo sostenible, cabe señalar los dos siguientes: practicar una pedagogía transformadora orientada a la acción que involucre a los alumnos en procesos de pensamiento y actuación participativos, sistémicos, creativos e innovadores en el contexto de las comunidades locales y de su propia vida y actuar como agentes de cambio en un proceso de aprendizaje organizacional que avance hacia el desarrollo sostenible.

En este contexto, desde la Facultad de Ciencias Sociales y de la Comunicación de la Universidad Europea de Madrid, un grupo de profesores hemos considerado de interés abrir una línea de investigación enmarcada dentro de un proyecto de investigación-acción que contemple la inclusión educativa y la sostenibilidad con la temática de los ODS y que brinde a los docentes la oportunidad de aplicar estos objetivos en el aula.

La metodología utilizada es flipped classroom o aula invertida, ya que se ha considerado la metodología adecuada para permitir que el alumno profundice en los problemas a los que se enfrenta el planeta, sea consciente de los mismos y, en consecuencia, adquiera las competencias necesarias que le permitan aportar soluciones.

La investigación-acción recoge una tendencia a conceptualizar la investigación educativa en términos participativos, representando un proceso a través del cual los sujetos investigados son verdaderos coinvestigadores que participan, de forma activa, en el planteamiento del problema (que ha de ser algo que les afecte o interese) y en la información que debe obtenerse (que marca el curso de la investigación). El investigador actúa esencialmente como un facilitador del proceso y un recurso disponible para ser consultado (Martínez, 2000).

En definitiva, se inicia un proyecto de investigación-acción que permita mantener los vínculos con la metodología flipped classroom y conduzca a alcanzar los objetivos planteados en relación con los ODS. Para ello, en este trabajo, en primera instancia se profundiza en la metodología aplicada. En segundo lugar se desarrollan los objetivos de la investigación, describiéndose posteriormente las actividades realizadas por los docentes así como su evaluación y resultados obtenidos. Finalmente se sintetizan las principales conclusiones obtenidas y las futuras líneas de investigación abiertas.

\section{Metodología}

Flipped classrom nació de una idea basada en la simple observación que sirvió para cambiar los enfoques educativos. Aaron Sans, profesor de Woodland Park High School (Colorado, EE. UU.), advirtió que los alumnos no requerían que el profesor esté físicamente con ellos en el aula para darles contenidos. Posteriormente, grabó las clases en vídeos de forma que 
los estudiantes las visualizaran como una tarea, quedando las clases presenciales para ayudar a los estudiantes con los conceptos que no entendieran (Bergman \& Sams, 2012). Las diferencias entre este innovador modelo pedagógico sustentando en la construcción del aprendizaje y el método tradicional basado en la instrucción directa para impartir clase quedan plasmadas en la tabla 1.

Tabla 1. Comparación de algunos elementos de la enseñanza basada en el profesor y "mediada" por el profesor en función de las dimensiones que se indican

\begin{tabular}{|c|c|c|}
\hline & Instrucción "directa" & Construcción \\
\hline Actividad & $\begin{array}{c}\text { Centrada en el profesor } \\
\text { Didáctica }\end{array}$ & $\begin{array}{c}\text { Centrada en el alumno } \\
\text { Interactiva }\end{array}$ \\
\hline Rol del profesor & $\begin{array}{c}\text { Transmisor de hechos, datos, etc. } \\
\text { Siempre experto }\end{array}$ & $\begin{array}{c}\text { Colaborador } \\
\text { Algunas veces aprendiz }\end{array}$ \\
\hline Rol del alumno & $\begin{array}{c}\text { Oyente } \\
\text { Ciempre aprendiz }\end{array}$ & $\begin{array}{c}\text { Colaborador } \\
\text { Algunas veces experto }\end{array}$ \\
\hline Énfasis instruccional & Hechos \\
Memorización & Relaciones \\
\hline
\end{tabular}

Fuente: Touron, Santiago y Díez (2014)

Flipped classroom permite a los profesores aprovechar la tecnología para aumentar la interacción con los estudiantes (Bergman \& Sams, 2012). Actualmente este modelo didáctico, apoyado en las nuevas tecnologías de la información, se aplica en diversos ámbitos de conocimiento de la enseñanza superior reflejados en la figura 1 a partir del trabajo realizado por Sola, Aznar, Romero y Rodríguez-García (2019), en el que se analizan los estudios indexados en las bases de datos de Scopus y Wos.

Figura 1. Materias objeto de estudio en las que se aplica el método flipped classroom

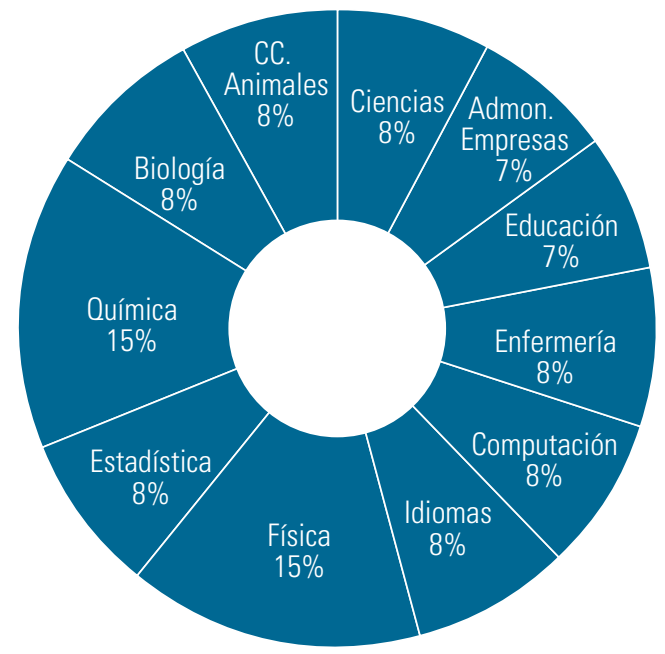


La variabilidad de las materias objeto de estudio pone de manifiesto la inquietud docente por conseguir que se pueda mejorar el aprendizaje de los alumnos. Según Aguilera, Manzano, Martínez, Lozano y Casiano (2017) esta metodología puede resultar adecuada en distintas áreas de conocimiento y cada vez son más los docentes que la están incorporando.

El aula invertida se ha convertido en un enfoque pedagógico cada vez más utilizado para la enseñanza y el aprendizaje en el ámbito de la enseñanza superior. Los estudios realizados por la comunidad científica avalan los beneficios de su implementación. Los datos de trabajos como el de Mattis (2015) indican que el rendimiento académico mejora. Los resultados de la investigación de Crimmins \& Midkiff (2017) sugieren que, si bien el aprendizaje mejoró los resultados en general para todos los estudiantes que lo recibieron, aquellos con los niveles más bajos de rendimiento académico experimentaron el mayor aumento. La percepción de los estudiantes sobre los beneficios de este modelo educativo indica un mayor nivel de satisfacción (Cheng, Ritzhaupt, \& Antoneko, 2018). La pedagogía del aula invertida es más eficaz que las conferencias tradicionales para mejorar el conocimiento teórico (Hu, Gao, Ye, Ni, Jiang, \& Jiang, 2018), permitiendo al alumno comprender la utilidad de los contenidos teóricos para cuando tenga la oportunidad de aplicarlos en el futuro (Matzumura, Gutiérrez, Zamudio, y Zavala, 2018).

En el polo opuesto, el método pedagógico conlleva que puedan surgir algunos inconvenientes. El estudiante acostumbrado a aprender de forma pasiva, puede presentar resistencia a aprender de forma activa negándose a dejar su zona de confort (Aguilera et al., 2017), o puede no gestionar bien el tiempo para estudiar por su cuenta los contenidos teóricos (Matzumura et al., 2018). Por otro lado, la aplicación de flipped classroom puede suponer un esfuerzo adicional para el profesor (Jordán, Pérez, \& Sanabria, 2014), ya que deberá crear materiales que se adecuen a este modelo pedagógico, reorganizar la planificación docente para clases presenciales y no presenciales y realizar un seguimiento del aprendizaje de los alumnos. En definitiva, conocer que el método puede conllevar algunas desventajas permite que se intenten erradicar para poder llevar a buen fin este proyecto de investigación.

\section{Planteamiento de objetivos}

El objetivo principal del proyecto de investigación-acción es incorporar, en las áreas de conocimiento de los futuros egresados, el mensaje contenido en la Agenda 2030 de Naciones Unidas con el propósito de que lo interioricen y, así, favorecer que puedan incorporarlo a su actividad profesional o laboral. Se han planteado los siguientes objetivos específicos:

» Primer objetivo específico: determinar si los alumnos desarrollan competencias para la investigación y la valoración crítica.

» Segundo objetivo específico: conocer si los estudiantes adquieren capacidades que pongan en valor el pensamiento crítico en relación a diferentes actuaciones en los ámbitos político, social, empresarial y jurídico.

» Tercer objetivo específico: determinar si los alumnos desarrollan competencias en relación directa con la responsabilidad social empresarial, la ética en los negocios y la actividad profesional.
Flipped classrom nació de una idea basada en la simple observación que sirvió para cambiar los enfoques educativos: los alumnos no requerían que el profesor esté físicamente con ellos en el aula para darles contenidos 


\section{Descripción de la actividad}

El equipo investigador está formado por profesores que imparten docencia en tres áreas de conocimiento adscritas a la Facultad de Ciencias Sociales y de la Comunicación: economía y empresa, comunicación y ciencias jurídicas y políticas. Esta diversidad intelectual resulta muy enriquecedora en un proyecto de investigación con carácter multidisciplinar. De hecho, se ha aplicado en cinco asignaturas diferentes, que, a su vez, se imparten en cuatro grados distintos.

La labor del profesor ha sido coordinar el aprendizaje, fomentar el análisis crítico y el trabajo autónomo. Las actividades se han implementado en el primer semestre del curso 2018/2019 en asignaturas incluidas en los planes de estudio de grados pertenecientes a la Facultad de Ciencias Sociales y de la Comunicación de la Universidad Europea de Madrid (tabla 2).

Finalizadas las actividades en cada grupo, se ha facilitado a los estudiantes una encuesta de carácter voluntario que ha servido de apoyo a las conclusiones de la investigación final.

Tabla 2. Asignaturas y grados de implementación de la actividad

\begin{tabular}{|c|c|}
\hline Asignatura & Grado \\
\hline Eficacia y Desarrollo Profesional & Administración y Dirección de Empresas \\
\hline Geografía Humana, Económica y Política & Relaciones Internacionales \\
\hline Análisis Financiero de Marketing & Publicidad \\
\hline Contabilidad de Costes & Administración y Dirección de Empresas \\
\hline Análisis de Estados Contables & Dirección y Creación de Empresas \\
\hline
\end{tabular}

Fuente: elaboración propia

La muestra analizada en este trabajo, está compuesta por los 166 alumnos (tabla 3) que durante el año académico 2018-2019 han cursado las asignaturas (tabla 2) en las que se ha implementado la actividad.

\section{Tabla 3. Tamaño de la muestra}

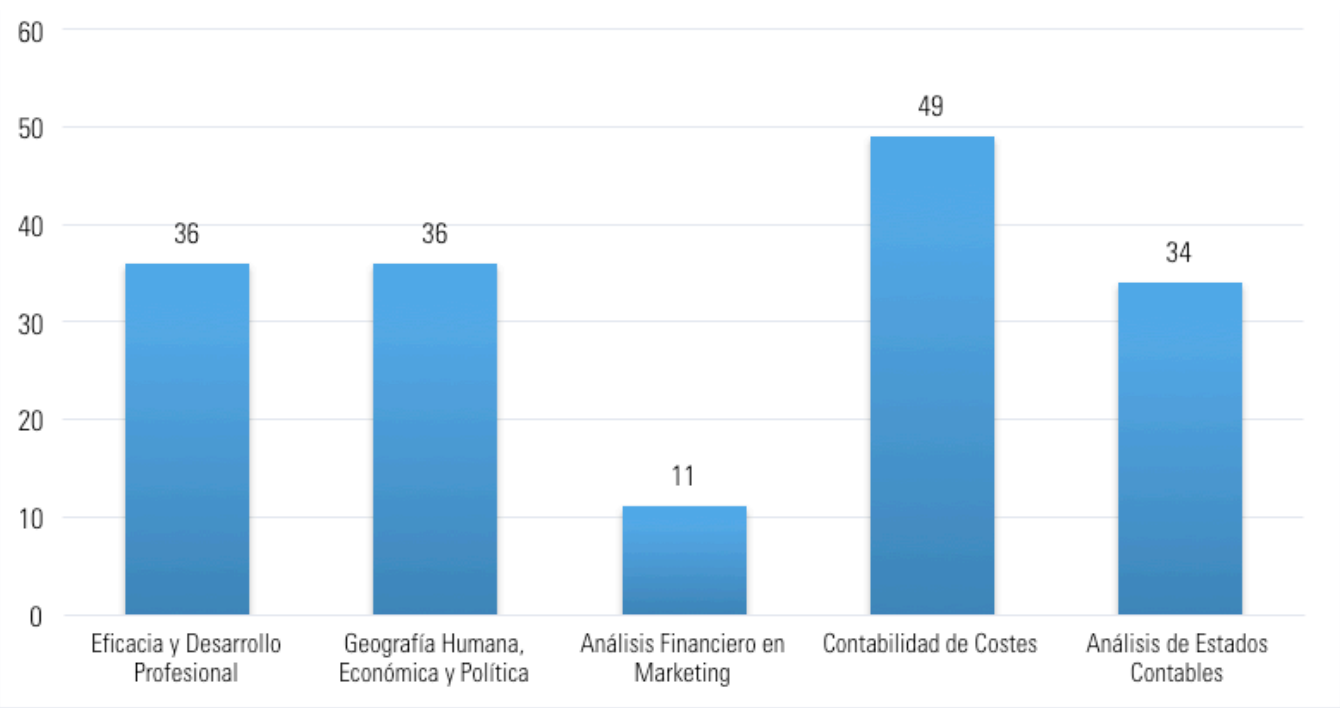

Fuente: elaboración propia 


\subsection{Eficacia y Desarrollo Profesional}

La actividad fue desarrollada en la asignatura Eficacia y Desarrollo Profesional, del primer curso del grado de Administración y Dirección de Empresa. Se realizó en los dos campus de la Universidad Europea de Madrid y contó con la participación de 20 alumnos en el grupo del campus de Alcobendas y de 16 alumnos en el grupo del campus de Villaviciosa de Odón.

El objetivo de la actividad era, principalmente, ayudar en el avance del consumo responsable de los estudiantes. Los alumnos analizaron las empresas de ropa más conocidas y más consumidas por ellos. De esta manera, conocieron el compromiso de las marcas que les visten con la sostenibilidad del planeta y con ello poder evaluarlas a la hora de su decisión de compra final.

Previamente al inicio de la actividad, se explicaron los aspectos más relevantes de los 17 Objetivos para el Desarrollo Sostenible. Después de las dos sesiones teóricas, en las que se combinaron la clase magistral con la visión de vídeos de apoyo, los estudiantes formaron grupos de un número máximo de 6 personas para trabajar la práctica de la actividad.

Cada grupo seleccionó una de las marcas de ropa actuales para, a través de su estudio, identificar cuál de los 17 ODS eran aplicados en las acciones empresariales de cada una de las marcas.

La actividad tuvo un desarrollo externo y otro interno en lo que al aula se refiere. Los estudiantes trabajaban durante la semana, organizados ellos mismos, en la actividad.

El profesor coordinaba la adquisición de conocimiento en cada una de sus actuaciones de estudio para después, durante una hora de las 4 que duraba la clase habitual de la asignatura, presentaran sus avances y plantearan las dudas para seguir implementando la práctica.

Al finalizar el semestre, los estudiantes presentaron en el aula el resultado de su estudio, que incluía una valoración personal referenciada al conocimiento y actuación de los Objetivos de Desarrollo Sostenible en las acciones empresariales de la marca elegida.

\subsection{Geografía Humana, Económica y Política}

La actividad se implementó en dos grupos de la asignatura Geografía Humana, Económica y Política perteneciente al primer curso del grado de Relaciones Internacionales. En el primer grupo, donde se imparte la asignatura en castellano, participaron 25 alumnos, mientras en el segundo grupo, donde se imparte en inglés, participaron 11 alumnos. La actividad, inserta en el tema 4 -Geografía Económica y Desarrollo - tenía por objetivo que los alumnos conocieran los ODS y reconocieran el objetivo número 5, sobre igualdad de género, como base imprescindible para la construcción de un mundo más justo.

Como iniciación a la actividad, la profesora realizó una sesión introductoria sobre la responsabilidad social corporativa y ofreció una revisión general de los ODS enfatizando el ODS 5. Se discutió en clase la problemática del matrimonio infantil, medida por el objetivo 5.3, a la vez que los alumnos reflexionaron sobre las implicaciones y consecuencias generales que dicha situación conlleva para las propias niñas, para sus familias, sus comunidades e incluso sus países. En la siguiente sesión los alumnos, en equipos de dos a tres miembros, eligieron un país con alta incidencia en matrimonio infantil. Cada equipo realizó una breve investigación sobre ese país basada en los materiales facilitados a los alumnos procedentes de fuentes oficiales del propio país, UNICEF y ONG. Todas las presentaciones debían contener los siguientes datos: los indicadores de Naciones Unidas sobre matrimonio infantil; los avances y retrocesos del país
Los alumnos analizaron las empresas de ropa más conocidas y más consumidas por ellos. De esta manera, conocieron el compromiso de las marcas que les visten con la sostenibilidad del planeta 
asignado en los últimos años, y las medidas y programas emprendidos por el gobierno, ONG, la ONU y demás organismos internacionales para acabar con el problema.

Finalmente, en una tercera sesión cada equipo diseñó una infografía utilizando la herramienta virtual Picktochart que reflejaba la situación del matrimonio infantil en el país asignado y, usándola como base a su explicación, presentó oralmente sus hallazgos a la clase. Con la actividad los alumnos explicaron cómo la eliminación del matrimonio infantil afectaría positivamente a la consecución de los objetivos 1 (Fin de la pobreza), 2 (Hambre cero) 3 (Salud y Bienestar), 4 (Educación de Calidad), 8 (Trabajo decente y Crecimiento Económico), 10 (Reducción de las Desigualdades) y 16 (Paz, Justicia e instituciones sólidas).

\subsection{Análisis Financiero de Marketing}

La actividad se realizó en la asignatura de Análisis Financiero de Marketing del grado en Publicidad, con la participación de un total de 11 alumnos. La asignatura se imparte en el 4. ${ }^{\circ}$ curso del grado, después de que los alumnos hayan recibido una amplia formación en la gestión de cuentas de las agencias de comunicación.

La actividad se ha implementado para que los alumnos adquieran competencias que les permitan incorporar criterios sostenibles en las acciones de comunicación que hayan de realizar en su futura vida laboral o profesional.

El conocimiento de la asignatura Análisis Financiero de Marketing permite a los estudiantes comprender el resultado previsto por la realización de una campaña o evento, así como analizar la información financiera de la empresa cliente, mediante ratios que permitan acreditar su solvencia y rentabilidad. La actividad se ha enfocado a reforzar los conocimientos financieros adquiridos con la incorporación de los criterios sostenibles recogidos en la información no financiera de las empresas.

En una sesión inicial, siguiendo la metodología flipped classroom, se ha facilitado a los alumnos materiales de estudio consistentes en legislación, páginas web y vídeos que sirven como soporte para conocer la información no financiera divulgada por las compañías cotizadas y su relación con los ODS de Naciones Unidas. En una segunda sesión se ha planteado un debate en el aula, que ha servido como referencia para el comienzo del trabajo de investigación.

El trabajo se ha realizado en grupos formados por tres alumnos, a los que se les ha planteado una simulación consistente en suponer que forman parte de un equipo de dirección de cuentas de una agencia de publicidad o de una agencia de marketing. El equipo ha captado un nuevo cliente (la compañía cotizada analizada) y en función de la actividad de la firma han elaborado una campaña de publicidad o un evento que ha incluido criterios relacionados con las tres vertientes de la sostenibilidad: sociales, medioambientales y económicas.

Por último, los grupos de trabajo han presentado en el aula el informe que han elaborado con las líneas generales de la campaña o del evento que se va a presentar a la empresa cliente, las cuestiones sobre sostenibilidad que se han incorporado y cómo se ha contribuido a la consecución de los ODS.

\subsection{Contabilidad de Costes}

El trabajo se llevó a cabo en dos grupos de la asignatura de Contabilidad de Costes, impartida en el 3. ${ }^{\circ}$ curso del grado de ADE (Administración y Dirección de Empresas), con un total de 24 alumnos en el primer grupo y 25 alumnos el segundo. 
La asignatura de Contabilidad de Costes tiene como objetivo que los alumnos sepan calcular los costes de los productos o servicios que presta una empresa y conozcan los diferentes métodos y modelos que existen para conseguirlo. Para ello es fundamental que los alumnos aprendan a analizar los procesos productivos y de prestación de servicios, ya que en función de estos se decidirá la idoneidad del método de cálculo a seleccionar. Por esta razón este trabajo se ha enfocado a los procesos productivos y su objetivo ha sido que los alumnos investigaran sobre empresas reales que desarrollaran procesos sostenibles y que avanzaran en uno o varios ODS.

Siguiendo la metodología aula invertida, el trabajo se ha planteado inicialmente entregando a los alumnos material (documentos, vídeos, webs) sobre sostenibilidad empresarial y sobre los ODS. Todo ello fue posteriormente debatido en el aula y les sirvió como marco de referencia para llevar a cabo su investigación.

A partir de aquí, los alumnos formaron grupos de trabajo y tuvieron que localizar una empresa real que llevara a cabo un proceso de producción o de prestación de servicios que consideraran sostenible, bien por las materias o suministros que utilizaba, o por las características del proceso, por el producto o servicio que ofertaba, o por su relación con el entorno social o medioambiental. Adicionalmente debían analizar a cuál o cuáles de los ODS contribuía la compañía en función del análisis precedente.

Finalmente, cada grupo de trabajo presentó en clase la investigación realizada, destacando los elementos clave utilizados para la selección de la empresa analizada y su compromiso con la sostenibilidad. A partir de aquí se abrió un debate sobre las diferentes formas de contribuir de cada compañía a la consecución de los ODS.

\subsection{Análisis de Estados Contables}

El trabajo se ha llevado a cabo con alumnos del grado en Dirección y Creación de Empresas (DICRE), en la asignatura de Análisis de Estados Contables tanto en el campus de Villaviciosa de Odón, donde participaron 26 alumnos, como en el campus de Alcobendas, con 8 alumnos. Esta materia se imparte en el $4{ }^{\circ}$ curso del grado, cuando los alumnos han recibido una amplia formación en contabilidad que les permite analizar e interpretar tanto la información financiera como la información no financiera divulgada por las compañías cotizadas.

La divulgación de información no financiera pone de manifiesto la responsabilidad social de las empresas o responsabilidad social corporativa (RSC) mediante la integración de las preocupaciones sociales y medioambientales en sus operaciones empresariales y en las relaciones con sus interlocutores o grupos de interés. La responsabilidad social de las empresas se ha convertido, a nivel global, en un concepto cada vez más importante que forma parte de la sostenibilidad (Comisión Europea, 2006).

En este contexto, el objetivo del trabajo realizado ha sido que los alumnos a través del análisis de la información no financiera divulgada por las compañías cotizadas en el índice IBEX 35 de la Bolsa de Madrid, puedan conocer cómo abordan estas entidades las necesidades de la sociedad y cómo se sustentan en criterios sostenibles para contribuir a los ODS de la Agenda 2030.

Para ello, se entregó a los alumnos materiales en forma de vídeos y artículos con información sobre RSC, sostenibilidad y ODS aplicados al mundo de la empresa. Todos estos materiales debían servir para poder determinar de forma razonada el motivo que les había llevado a seleccionar la entidad objeto de estudio, analizar la estructura de la información divulgada sobre
La divulgación de información no financiera pone de manifiesto la responsabilidad social de las empresas o responsabilidad social corporativa (RSC) mediante la integración de las preocupaciones sociales $y$ medioambientales 
sostenibilidad, conocer las directrices o normas en las que se sustentaba dicha información y, por último, determinar el sistema de verificación de la información. De esta forma, los alumnos obtuvieron los elementos necesarios para comprender el trabajo que debían realizar. Por último, siempre en función del análisis precedente, los alumnos debían mostrar una opinión crítica sobre la información proporcionada por la compañía e indicar su contribución a los ODS.

A continuación, los alumnos, se organizaron en grupos y seleccionaron una empresa cotizada. Los trabajos realizados fueron presentados por los alumnos en clase y se llevó a cabo un interesante intercambio de opiniones sobre las conclusiones obtenidas. Entre las cuestiones que fueron motivo de debate cabe destacar el interés por la sostenibilidad y los ODS que se observó en todas las compañías analizadas, la existencia de sistemas de información estructurados sobre sostenibilidad y de verificación de la misma, así como la relevancia de los ODS entre los objetivos de estas compañías.

\section{Evaluación}

En esta investigación se ha considerado que los alumnos deben adquirir competencias que les permitan conseguir la formación necesaria para interiorizar el mensaje contenido en la Agenda 2030. Las competencias trabajadas durante la actividad han sido de gran calado académico puesto que así lo requiere el ADN de la Universidad Europea de Madrid y, asimismo, forma parte de la propia idiosincrasia de esta investigación. Además del conocimiento y del compromiso reglado a la aportación académica, los estudiantes que han participado han desarrollado implícitamente y explícitamente una serie de competencias que les han permitido alcanzar los objetivos previamente planteados (tabla 4).

Tabla 4. Objetivos y competencias

\begin{tabular}{|c|c|}
\hline Objetivos & Competencias \\
\hline $\begin{array}{c}\text { Desarrollar competencias para la investigación y la } \\
\text { valoración crítica }\end{array}$ & $\begin{array}{c}\text { Compromiso } \\
\text { Capacidad de gestión y planificación } \\
\text { Habilidades comunicativas }\end{array}$ \\
\hline $\begin{array}{c}\text { Poner en valor el pensamiento crítico en relación a } \\
\text { diferentes actuaciones en los ámbitos político, social, } \\
\text { empresarial y jurídico }\end{array}$ & $\begin{array}{c}\text { Capacidad de reunir e interpretar datos relevantes que permitan } \\
\text { emitir juicios que incluyan una reflexión sobre temas relevantes de } \\
\text { índole social, científica o ética }\end{array}$ \\
\hline $\begin{array}{c}\text { Desarrollar competencias en relación directa con la } \\
\text { responsabilidad social empresarial, la ética en los negocios } \\
\text { y en la actividad profesional }\end{array}$ & Conciencia de los valores éticos \\
\hline
\end{tabular}

Fuente: elaboración propia

Las competencias establecidas para lograr el primer objetivo específico, investigación y valoración crítica son el compromiso, la capacidad de gestión y planificación y las habilidades comunicativas. Con la primera se ha valorado el grado de responsabilidad compartida del estudiante con las tareas encomendadas dado que la metodología del aula invertida no ha requerido una supervisión exhaustiva por parte de los profesores.

Asimismo, los estudiantes han tenido que establecer un orden de prioridades y un reparto funcional de los recursos. Cualquier cometido que sea mínimamente complejo y se divida en tareas requiere establecer un orden de prioridades y un reparto funcional de los recursos que ponga de manifiesto la capacidad de gestión y planificación. 
Los estudiantes han tenido que exponer los resultados de su investigación tanto en comunicación escrita como verbal. La competencia relacionada con las habilidades comunicativas de los estudiantes resulta fundamental para comprender la coherencia de los conocimientos adquiridos y expuestos durante sus intervenciones. Dentro de esta competencia se incluye la comunicación interpersonal, ya que se ha valorado el buen ambiente en los equipos de trabajo y el buen manejo de la información intergrupal.

Para alcanzar el segundo objetivo específico y poner en valor el pensamiento crítico en relación a diferentes actuaciones en los ámbitos políticos, sociales, empresariales y jurídicos, se ha considerado de interés desarrollar la capacidad de reunir e interpretar datos relevantes que, dependiendo del área de estudio, permitan emitir juicios que incluyan una reflexión sobre temas esenciales de índole social, científica o ética.

Por último, para lograr el tercer objetivo específico, la competencia denominada conciencia de los valores éticos se considera imprescindible para este tipo de investigación, ya que permite desarrollar competencias relacionadas con la responsabilidad social empresarial, la ética en los negocios y la ética profesional. Esta competencia impulsa la capacidad para pensar y actuar según principios universales basados en el valor de la persona que se dirigen a su pleno crecimiento y que conllevan el compromiso con el desarrollo sostenible en sus tres vertientes: social, medioambiental y económica.

En este contexto pedagógico, se ha utilizado la rúbrica como herramienta de evaluación, debido a que, como destacan Buján, Rekalde y Aramedi (2011), entre otros autores, las rúbricas son una herramienta excelente para la evaluación, no solo de contenidos, sino también de competencias. Constituyen una forma de estandarizar la evaluación en relación a una serie de criterios específicos para, de esta forma, convertirla en un proceso más sencillo y transparente. Adicionalmente, resultan herramientas ideales para reducir la subjetividad en la corrección de trabajos, ya que clarifican el proceso de evaluación recogiendo los criterios de los diferentes niveles alcanzados y aportan a los estudiantes una interesante retroalimentación del trabajo realizado. En la rúbrica desarrollada se han definido tres bloques de competencias a desarrollar en función de los objetivos previamente establecidos y se han considerado siete indicadores que permiten mostrar las evidencias de la eficacia del proceso de aprendizaje en función de la adquisición de competencias. Asimismo, para cada uno de los indicadores, se han planteado cuatro niveles de dominio que, de forma creciente, reflejan el grado de conocimiento adquirido (tabla 5).

\section{Los estudiantes}

han tenido que

exponer los

resultados de su

investigación tanto

en comunicación

escrita como

verbal. La

competencia

relacionada con

las habilidades

comunicativas de

los estudiantes

resulta fundamental

para comprender

la coherencia de

los conocimientos

adquinidos 
Tabla 5. Rúbrica de evaluación de las actividades

\begin{tabular}{|c|c|c|c|c|c|c|}
\hline \multicolumn{6}{|c|}{ Rúbrica de evaluación ODS } & \\
\hline \multirow{3}{*}{$\begin{array}{l}\text { OBJETIVOS Y } \\
\text { COMPETENCIAS }\end{array}$} & \multirow{3}{*}{ Indicadores } & \multirow{3}{*}{ PESO } & \multicolumn{4}{|c|}{ Nivel de dominio } \\
\hline & & & Nivel I. Insatisfactorio & Nivel II. Mejorable & Nivel III. Satisfactorio & Nivel IV. Excelente \\
\hline & & & $(0-4,9)$ & (5,0 - 6,99 puntos) & (7,0 - 8,9 puntos) & (9,0 - 10 puntos) \\
\hline \multirow[t]{3}{*}{$\begin{array}{c}\text { Primer objetivo: } \\
\text { competencias } \\
\text { para la } \\
\text { investigación y } \\
\text { valoración crítica }\end{array}$} & $\begin{array}{l}\text { Compromiso. } \\
\text { Grado de } \\
\text { responsabilidad } \\
\text { compartida }\end{array}$ & $13 \%$ & $\begin{array}{l}\text { No se percibe un reparto } \\
\text { equitativo de las tareas. } \\
\text { El trabajo se presenta } \\
\text { descuidado y desordenado }\end{array}$ & $\begin{array}{l}\text { El grado de responsabilidad } \\
\text { compartida no parece el } \\
\text { mismo entre los miembros } \\
\text { del equipo. El trabajo se } \\
\text { presenta sin portada, } \\
\text { identificación del trabajo } \\
\text { y del grupo, índice de } \\
\text { contenidos y debidamente } \\
\text { estructurado y ordenado }\end{array}$ & $\begin{array}{l}\text { El grado de responsabilidad } \\
\text { compartida con las } \\
\text { tareas encomendadas es } \\
\text { adecuado y equitativo. } \\
\text { Se aporta una correcta } \\
\text { redacción y extensión y } \\
\text { está bien estructurado y } \\
\text { organizado }\end{array}$ & $\begin{array}{l}\text { Fuerte compromiso de } \\
\text { todos los miembros con } \\
\text { las tareas encomendadas. } \\
\text { El trabajo es de una } \\
\text { presentación adecuada, } \\
\text { correcta y ordenada }\end{array}$ \\
\hline & $\begin{array}{l}\text { Capacidad } \\
\text { de gestión. } \\
\text { Priorización } \\
\text { y reparto de } \\
\text { recursos }\end{array}$ & $13 \%$ & $\begin{array}{l}\text { No utiliza recursos } \\
\text { académicos y no } \\
\text { académicos adecuados o } \\
\text { relevantes }\end{array}$ & $\begin{array}{l}\text { Utiliza recursos académicos } \\
\text { y no académicos adecuados } \\
\text { o relevantes, limitándose } \\
\text { a enumerarlos, sin } \\
\text { particularizar al caso de } \\
\text { análisis }\end{array}$ & $\begin{array}{c}\text { Utiliza recursos académicos } \\
\text { y no académicos adecuados } \\
\text { o relevantes y los utiliza } \\
\text { para realizar un análisis } \\
\text { superficial de su objeto de } \\
\text { estudio }\end{array}$ & $\begin{array}{c}\text { Utiliza recursos } \\
\text { adecuados o relevantes; } \\
\text { los utiliza para analizar } \\
\text { correctamente el objeto de } \\
\text { estudio }\end{array}$ \\
\hline & $\begin{array}{l}\text { Exposición } \\
\text { escrita y oral de } \\
\text { resultados }\end{array}$ & $14 \%$ & $\begin{array}{l}\text { La forma de explicar la } \\
\text { información no es nada } \\
\text { clara. No se produce } \\
\text { contacto visual con la } \\
\text { audiencia y su postura es } \\
\text { inadecuada }\end{array}$ & $\begin{array}{l}\text { La forma de explicar la } \\
\text { información es poco clara } \\
\text { y precisa. Rara vez su } \\
\text { postura es correcta y hay } \\
\text { poco contacto visual con la } \\
\text { audiencia }\end{array}$ & $\begin{array}{l}\text { La mayor parte de la } \\
\text { información se expresa } \\
\text { con precisión y claridad. } \\
\text { Normalmente tiene una } \\
\text { postura adecuada y existe } \\
\text { el contacto visual con la } \\
\text { audiencia }\end{array}$ & $\begin{array}{l}\text { En toda la presentación } \\
\text { se expresa la información } \\
\text { con precisión y claridad, } \\
\text { ayudándose de ejemplos } \\
\text { para mejorar la comprensión } \\
\text { de las ideas. En todo } \\
\text { momento se mantiene una } \\
\text { postura correcta y existe el } \\
\text { contacto visual }\end{array}$ \\
\hline \multirow{2}{*}{$\begin{array}{l}\text { Segundo objetivo: } \\
\text { competencias } \\
\text { sobre } \\
\text { pensamiento } \\
\text { crítico en } \\
\text { los ámbitos } \\
\text { político, social, } \\
\text { empresarial y } \\
\text { jurídico }\end{array}$} & $\begin{array}{l}\text { Capacidad de } \\
\text { reunir e interpretar } \\
\text { datos relevantes }\end{array}$ & $15 \%$ & $\begin{array}{l}\text { No es capaz de buscar, } \\
\text { recopilar, sintetizar e } \\
\text { integrar información } \\
\text { necesaria para abordar el } \\
\text { problema }\end{array}$ & $\begin{array}{c}\text { Busca, recopila, sintetiza e } \\
\text { integra información básica } \\
\text { necesaria para abordar el } \\
\text { problema }\end{array}$ & $\begin{array}{l}\text { Busca, recopila, sintetiza } \\
\text { e integra información } \\
\text { compleja o ambigua } \\
\text { necesaria para abordar el } \\
\text { problema }\end{array}$ & $\begin{array}{c}\text { Busca, recopila, sintetiza } \\
\text { e integra información muy } \\
\text { compleja o muy ambigua } \\
\text { necesaria para abordar el } \\
\text { problema }\end{array}$ \\
\hline & $\begin{array}{l}\text { Capacidad de } \\
\text { emitir juicios } \\
\text { que incluyan } \\
\text { reflexiones sobre } \\
\text { temas sociales, } \\
\text { científicos o éticos }\end{array}$ & $15 \%$ & $\begin{array}{l}\text { Analiza el problema } \\
\text { sin considerar varios } \\
\text { enfoques y desarrolla un } \\
\text { argumento que no tiene } \\
\text { coherencia o contiene } \\
\text { errores significativos en su } \\
\text { lógica. Tiende a basarse en } \\
\text { soluciones tradicionales }\end{array}$ & $\begin{array}{l}\text { Analiza el problema } \\
\text { considerando varios } \\
\text { enfoques y desarrolla } \\
\text { un argumento que tiene } \\
\text { sentido, pero no tiene } \\
\text { coherencia o contiene } \\
\text { errores significativos en } \\
\text { su lógica }\end{array}$ & $\begin{array}{l}\text { Analiza y explora el } \\
\text { problema considerando } \\
\text { distintas perspectivas, } \\
\text { enfoques alternativos } \\
\text { y evidencias antes de } \\
\text { formular una solución o } \\
\text { conclusión, desarrollando } \\
\text { un argumento lógico. Está } \\
\text { dispuesto a probar un } \\
\text { enfoque nuevo cuando } \\
\text { resuelve problemas }\end{array}$ & $\begin{array}{l}\text { Analiza y explora de forma } \\
\text { exhaustiva un problema } \\
\text { considerando distintas } \\
\text { perspectivas y evidencias } \\
\text { antes de formular una } \\
\text { solución o conclusión, } \\
\text { desarrollando un } \\
\text { argumento lógico, sólido y } \\
\text { completo. Identifica nuevas } \\
\text { maneras para resolver } \\
\text { problemas con el fin de } \\
\text { satisfacer las necesidades } \\
\text { de la situación }\end{array}$ \\
\hline \multirow[t]{2}{*}{$\begin{array}{l}\text { Tercer objetivo: } \\
\text { competencias } \\
\text { sobre la } \\
\text { responsabilidad } \\
\text { social } \\
\text { empresarial, } \\
\text { la ética en los } \\
\text { negocios y en } \\
\text { la actividad } \\
\text { profesional }\end{array}$} & $\begin{array}{l}\text { Analiza desde el } \\
\text { punto de vista ético } \\
\text { casos relacionados } \\
\text { con su actividad } \\
\text { profesional, } \\
\text { tomando } \\
\text { decisiones y } \\
\text { defendiéndolas } \\
\text { racionalmente }\end{array}$ & $15 \%$ & $\begin{array}{l}\text { No analiza desde el } \\
\text { punto de vista ético } \\
\text { casos relacionados con } \\
\text { su actividad profesional y } \\
\text { no es capaz de defender } \\
\text { racionalmente las } \\
\text { decisiones que toma }\end{array}$ & $\begin{array}{l}\text { Analiza desde el punto } \\
\text { de vista ético casos } \\
\text { relacionados con su } \\
\text { actividad profesional, } \\
\text { pero no es capaz de } \\
\text { defender racionalmente las } \\
\text { decisiones que toma }\end{array}$ & $\begin{array}{l}\text { Analiza desde el punto } \\
\text { de vista ético casos } \\
\text { relacionados con su } \\
\text { actividad profesional y } \\
\text { defiende racionalmente las } \\
\text { decisiones que toma }\end{array}$ & $\begin{array}{l}\text { Analiza en profundidad } \\
\text { casos relacionados con su } \\
\text { actividad profesional desde } \\
\text { el punto de vista ético, } \\
\text { toma las decisiones más } \\
\text { acertadas y las defiende } \\
\text { racionalmente de la } \\
\text { manera más adecuada }\end{array}$ \\
\hline & $\begin{array}{l}\text { Analiza un } \\
\text { problema de } \\
\text { responsabilidad } \\
\text { social mediante } \\
\text { argumentaciones } \\
\text { razonadas y } \\
\text { justificadas }\end{array}$ & $15 \%$ & $\begin{array}{l}\text { No es capaz de } \\
\text { localizar problemas de } \\
\text { responsabilidad social en } \\
\text { casos reales, por lo que no } \\
\text { opina sobre ellos }\end{array}$ & $\begin{array}{l}\text { Manifiesta su opinión } \\
\text { sobre problemas de } \\
\text { responsabilidad social, } \\
\text { pero no es capaz de } \\
\text { argumentar sus opiniones }\end{array}$ & $\begin{array}{l}\text { Manifiesta su opinión } \\
\text { sobre problemas de } \\
\text { responsabilidad social y } \\
\text { utiliza argumentos para } \\
\text { defender su postura }\end{array}$ & $\begin{array}{l}\text { Manifiesta su opinión } \\
\text { sobre problemas de } \\
\text { responsabilidad social } \\
\text { y utiliza argumentos } \\
\text { justificados sobre } \\
\text { sus posiciones, } \\
\text { transmitiéndolos de } \\
\text { manera adecuada a otros }\end{array}$ \\
\hline
\end{tabular}


Con referencia a los resultados de esta actividad, en términos de calificaciones académicas, el desglose de la nota media de las evaluaciones de la actividad implementada en cada uno de los grupos de las cinco asignaturas se muestra a continuación (tabla 6):

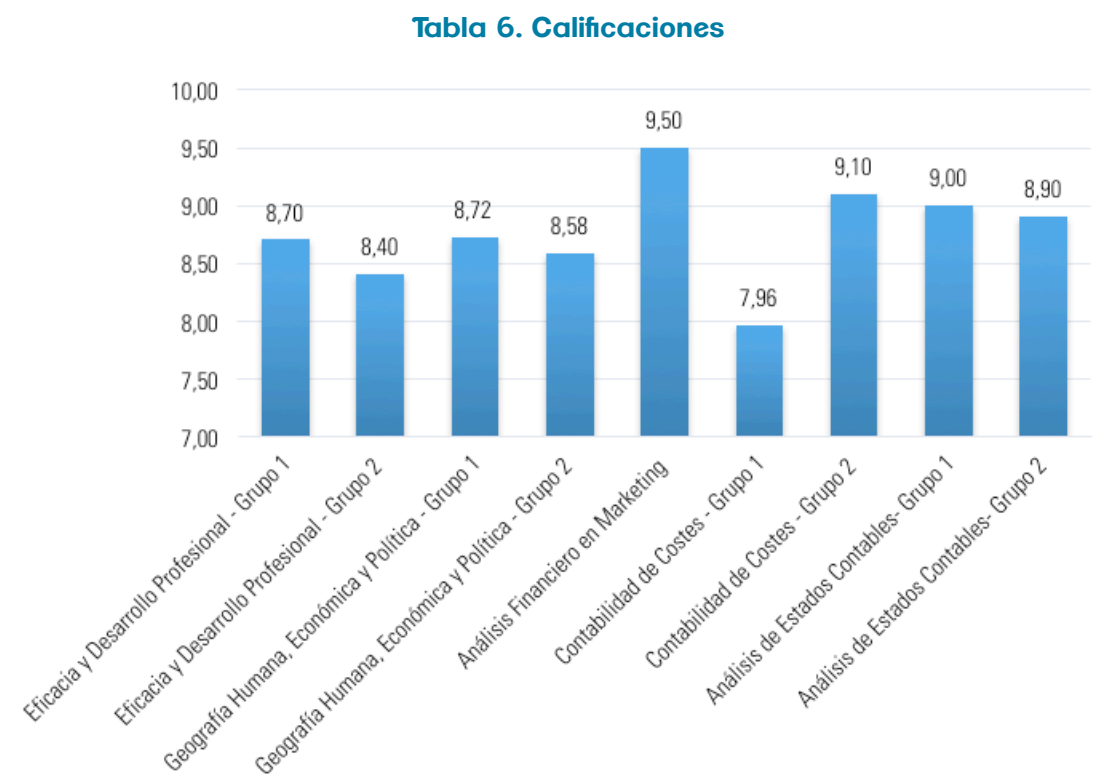

Fuente: elaboración propia

La calificación promedio de la actividad en las cinco asignaturas fue significativamente alta. Se atribuye esta nota al alto nivel de aceptación, trabajo y desempeño en esta actividad por parte de los estudiantes.

\section{Resultados}

Para conocer el grado de satisfacción de los estudiantes con las actividades desarrolladas se llevó a cabo una encuesta tras la finalización de las mismas. Para ello se realizó un cuestionario cerrado de 12 preguntas con una escala Likert con cinco opciones ( 1 totalmente en desacuerdo a 5 totalmente de acuerdo). El cuestionario se realizó online en las aulas y a través de la aplicación Formularios de Google.

Las encuestas se realizaron en el primer semestre del curso 2018-19 obteniendo una participación de 120 alumnos, de los que el 10\% corresponden a estudiantes de Análisis Financiero en Marketing, un 25\% a alumnos de Eficacia Personal y Profesional, el 26,6\% a estudiantes de la asignatura de Geografía Humana, Económica y Política, y el resto de los alumnos se repartió entre Análisis de Estados Contables y Contabilidad de Costes con un 19,2\% respectivamente.

Para analizar la satisfacción de los alumnos con las actividades realizadas se estructuraron en cinco bloques las cuestiones planteadas en la encuesta. El primer bloque se refería al grado de conocimiento previo de los alumnos sobre los ODS. En los siguientes bloques se plantearon cuestiones relativas a su opinión sobre la influencia de esta actividad en su conciencia ética y social, así como sobre su futuro profesional. A continuación, el cuarto bloque se componía de cuestiones relativas al desarrollo de sus competencias de aprendizaje y, por último, el quinto bloque se refería a su nivel de satisfacción con las actividades realizadas. 


\subsection{Conocimiento previo de los ODS}

La primera pregunta que se formuló fue referida al grado de conocimiento que poseían los alumnos sobre la temática de los ODS, previo a la actividad. Como puede observarse en la tabla 7 solo un $36,67 \%$ de los alumnos consideraron que tenían conocimientos sobre los ODS, siendo los alumnos de Contabilidad de Costes los que presentaban un mayor dominio del tema, seguidos de los estudiantes de Análisis de Estados Contables. Estas dos asignaturas se imparten en los últimos años del grado, por lo que parece razonable que hayan recibido más información sobre esta cuestión a lo largo de sus estudios que los alumnos de las otras materias impartidas en los primeros cursos del grado.

Tabla 7. Conocimientos previos sobre ODS

\begin{tabular}{|c|c|c|c|c|}
\hline $\begin{array}{c}\text { iConocía usted los Objetivos de Desarrollo } \\
\text { Sostenible de Naciones Unidas antes de Ilevar a } \\
\text { cabo esta actividad? }\end{array}$ & $\begin{array}{c}\text { Desacuerdo } \\
\%\end{array}$ & $\begin{array}{c}\text { Indiferente } \\
\%\end{array}$ & $\begin{array}{c}\text { Acuerdo } \\
\%\end{array}$ & $\begin{array}{c}\text { Total } \\
\%\end{array}$ \\
\hline Eficacia y Desarrollo Profesional & 66,67 & 6,67 & 26,67 & 100,00 \\
\hline Geografía Humana, Económica y Política & 62,50 & 12,50 & 25,00 & 100,00 \\
\hline Análisis Financiero en Marketing & 58,33 & 8,33 & 33,33 & 100,00 \\
\hline Contabilidad Costes & 21,74 & 13,04 & 65,22 & 100,00 \\
\hline Análisis Estados Contables & 26,09 & 34,78 & 39,13 & 100,00 \\
\hline Todas las asignaturas & 48,33 & 15,00 & 36,67 & 100,00 \\
\hline
\end{tabular}

Fuente: elaboración propia

\subsection{Influencia de la actividad en la conciencia ética y social del alumno}

Para conocer si los alumnos consideraban que la actividad había desarrollado su conciencia ética y social se les formuló tres cuestiones. La primera relacionada con su conocimiento sobre la responsabilidad social (tabla 8), la siguiente sobre el posible incremento de su compromiso social (tabla 9) y, por último, se les preguntó sobre la incidencia de la actividad en su sensibilidad hacia los problemas sociales y medioambientales (tabla 10). En todas ellas los alumnos mostraron de forma mayoritaria que las actividades habían contribuido a positivamente en las cuestiones planteadas, destacando el incremento del aprendizaje sobre la responsabilidad social corporativa (tabla 8), seguido del aumento de la conciencia social y medioambiental (tabla 10) y de la intensificación del compromiso social del alumno (tabla 9).

Tabla 8. Aprendizaje sobre RSC

\begin{tabular}{|c|c|c|c|c|}
\hline $\begin{array}{c}\text { ¿Considera que con esta actividad ha aumentado su nivel de } \\
\text { aprendizaje sobre la responsabilidad social corporativa? }\end{array}$ & $\begin{array}{c}\text { Desacuerdo } \\
\%\end{array}$ & $\begin{array}{c}\text { Indiferente } \\
\%\end{array}$ & $\begin{array}{c}\text { Acuerdo } \\
\%\end{array}$ & $\begin{array}{c}\text { Total } \\
\%\end{array}$ \\
\hline Eficacia y Desarrollo Profesional & 6,67 & 6,67 & 86,67 & 100,00 \\
\hline Geografía Humana, Económica y Política & 3,23 & 16,13 & 80,65 & 100,00 \\
\hline Análisis Financiero en Marketing & 0,00 & 8,33 & 91,67 & 100,00 \\
\hline Contabilidad Costes & 8,70 & 0,00 & 91,30 & 100,00 \\
\hline
\end{tabular}




\begin{tabular}{|c|c|c|c|c|}
\hline $\begin{array}{c}\text { ¿Considera que con esta actividad ha aumentado su nivel de } \\
\text { aprendizaje sobre la responsabilidad social corporativa? }\end{array}$ & $\begin{array}{c}\text { Desacuerdo } \\
\%\end{array}$ & $\begin{array}{c}\text { Indiferente } \\
\%\end{array}$ & $\begin{array}{c}\text { Acuerdo } \\
\%\end{array}$ & $\begin{array}{c}\text { Total } \\
\%\end{array}$ \\
\hline Análisis Estados Contables & 0,00 & 4,35 & 95,65 & 100,00 \\
\hline Todas las asignaturas & 4,20 & 7,56 & 88,24 & 100,00 \\
\hline
\end{tabular}

Fuente: elaboración propia

Tabla 9. Aumento del compromiso social

\begin{tabular}{|c|c|c|c|c|}
\hline $\begin{array}{c}\text { ¿Considera que con esta actividad se ha } \\
\text { incrementado su propio compromiso social? }\end{array}$ & $\begin{array}{c}\text { Desacuerdo } \\
\%\end{array}$ & $\begin{array}{c}\text { Indiferente } \\
\%\end{array}$ & $\begin{array}{c}\text { Acuerdo } \\
\%\end{array}$ & $\begin{array}{c}\text { Total } \\
\%\end{array}$ \\
\hline Eficacia y Desarrollo Profesional & 6,67 & 30,00 & 63,33 & 100,00 \\
\hline Geografía Humana, Económica y Política & 6,45 & 19,35 & 74,19 & 100,00 \\
\hline Análisis Financiero en Marketing & 18,18 & 27,27 & 54,55 & 100,00 \\
\hline Contabilidad Costes & 21,74 & 26,09 & 52,17 & 100,00 \\
\hline Análisis Estados Contables & 0,00 & 26,09 & 73,91 & 100,00 \\
\hline Todas las asignaturas & 9,32 & 25,42 & 65,25 & 100,00 \\
\hline
\end{tabular}

Fuente: elaboración propia

Tabla 10. Sensibilidad hacia problemas sociales y medioambientales

\begin{tabular}{|c|c|c|c|c|}
\hline $\begin{array}{c}\text { ¿Considera que con esta actividad se ha } \\
\text { incrementado su nivel de sensibilidad hacia los } \\
\text { problemas sociales o medioambientales? }\end{array}$ & $\begin{array}{c}\text { Desacuerdo } \\
\%\end{array}$ & $\begin{array}{c}\text { Indiferente } \\
\%\end{array}$ & $\begin{array}{c}\text { Acuerdo } \\
\%\end{array}$ & $\begin{array}{c}\text { Total } \\
\%\end{array}$ \\
\hline Eficacia y Desarrollo Profesional & 3,33 & 20,00 & 76,67 & 100,00 \\
\hline Geografía Humana, Económica y Política & 6,25 & 9,38 & 84,38 & 100,00 \\
\hline Análisis Financiero en Marketing & 8,33 & 33,33 & 58,33 & 100,00 \\
\hline Contabilidad Costes & 13,04 & 30,43 & 56,52 & 100,00 \\
\hline Análisis Estados Contables & 0,00 & 8,70 & 91,30 & 100,00 \\
\hline Todas las asignaturas & 5,83 & 18,33 & 75,83 & 100,00 \\
\hline
\end{tabular}

Fuente: elaboración propia

\subsection{Influencia de la actividad en las perspectivas y futuro profesional de los alumnos}

El tercer bloque de preguntas estaba referido a la incidencia de la actividad en el interés del desarrollo sostenible y responsable en su área profesional (tabla 11), así como su opinión en relación a la apertura de nuevas perspectivas profesionales gracias a la actividad (tabla 12). Los alumnos han considerado más importante el interés por el desarrollo sostenible despertado por la actividad, mientras que su posible aplicación en nuevas perspectivas profesionales se ha estimado menos interesante. 
Tabla 11. Interés profesional por el desarrollo sostenible

\begin{tabular}{|c|c|c|c|c|}
\hline $\begin{array}{c}\text { ¿Considera que esta actividad ha despertado su } \\
\text { interés por el desarrollo sostenible responsable } \\
\text { en su área profesional? }\end{array}$ & $\begin{array}{c}\text { Desacuerdo } \\
\%\end{array}$ & $\begin{array}{c}\text { Indiferente } \\
\%\end{array}$ & $\begin{array}{c}\text { Acuerdo } \\
\%\end{array}$ & $\begin{array}{c}\text { Total } \\
\%\end{array}$ \\
\hline Eficacia y Desarrollo Profesional & 6,67 & 23,33 & 70,00 & 100,00 \\
\hline Geografía Humana, Económica y Política & 0,00 & 21,88 & 78,13 & 100,00 \\
\hline Análisis Financiero en Marketing & 9,09 & 9,09 & 81,82 & 100,00 \\
\hline Contabilidad Costes & 8,70 & 17,39 & 73,91 & 100,00 \\
\hline Análisis Estados Contables & 0,00 & 26,09 & 73,91 & 100,00 \\
\hline Todas las asignaturas & 4,20 & 21,01 & 74,79 & 100,00 \\
\hline
\end{tabular}

Fuente: elaboración propia

Tabla 12. nuevas perspectivas de desarrollo profesional

\begin{tabular}{|c|c|c|c|c|}
\hline $\begin{array}{c}\text { ¿Considera que esta actividad le ha abierto } \\
\text { nuevas perspectivas para su futuro profesional? }\end{array}$ & $\begin{array}{c}\text { Desacuerdo } \\
\%\end{array}$ & $\begin{array}{c}\text { Indiferente } \\
\%\end{array}$ & $\begin{array}{c}\text { Acuerdo } \\
\%\end{array}$ & $\begin{array}{c}\text { Total } \\
\%\end{array}$ \\
\hline Eficacia y Desarrollo Profesional & 10,00 & 20,00 & 70,00 & 100,00 \\
\hline Geografía Humana, Económica y Política & 6,25 & 28,13 & 65,63 & 100,00 \\
\hline Análisis Financiero en Marketing & 8,33 & 25,00 & 66,67 & 100,00 \\
\hline Contabilidad Costes & 17,39 & 34,78 & 47,83 & 100,00 \\
\hline Análisis Estados Contables & 4,35 & 17,39 & 78,26 & 100,00 \\
\hline Todas las asignaturas & 9,17 & 25,00 & 65,83 & 100,00 \\
\hline
\end{tabular}

Fuente: elaboración propia

\subsection{Desarrollo de competencias de aprendizaje}

El cuarto bloque de cuestiones estaba relacionado con las competencias desarrolladas en la actividad: aprendizaje activo (tabla 13), trabajo en equipo (tabla 14), valores éticos (tabla 15) y comunicación (tabla 16). Los alumnos han valorado muy positivamente el desarrollo de estas cuatro competencias destacando la relativa a los valores éticos y el aprendizaje activo.

Tabla 13. Aprendizaje activo

\begin{tabular}{|c|c|c|c|c|}
\hline $\begin{array}{c}\text { ¿Considera que esta actividad promueve el } \\
\text { aprendizaje activo? }\end{array}$ & $\begin{array}{c}\text { Desacuerdo } \\
\%\end{array}$ & $\begin{array}{c}\text { Indiferente } \\
\%\end{array}$ & $\begin{array}{c}\text { Acuerdo } \\
\%\end{array}$ & $\begin{array}{c}\text { Total } \\
\%\end{array}$ \\
\hline Eficacia y Desarrollo Profesional & 3,33 & 20,00 & 76,67 & 100,00 \\
\hline Geografía Humana, Económica y Política & 0,00 & 18,75 & 81,25 & 100,00 \\
\hline Análisis Financiero en Marketing & 8,33 & 8,33 & 83,33 & 100,00 \\
\hline Contabilidad Costes & 4,35 & 13,04 & 82,61 & 100,00 \\
\hline Análisis Estados Contables & 0,00 & 26,09 & 73,91 & 100,00 \\
\hline Todas las asignaturas & 2,50 & 18,33 & 79,17 & 100,00 \\
\hline
\end{tabular}

Fuente: elaboración propia 
Tabla 14. Trabajo en equipo

\begin{tabular}{|c|c|c|c|c|}
\hline $\begin{array}{c}\text { ¿Considera que con esta actividad ha desarrollado } \\
\text { la competencia de trabajo en equipo? }\end{array}$ & $\begin{array}{c}\text { Desacuerdo } \\
\%\end{array}$ & $\begin{array}{c}\text { Indiferente } \\
\%\end{array}$ & $\begin{array}{c}\text { Acuerdo } \\
\%\end{array}$ & $\begin{array}{c}\text { Total } \\
\%\end{array}$ \\
\hline Eficacia y Desarrollo Profesional & 3,33 & 10,00 & 86,67 & 100,00 \\
\hline Geografía Humana, Económica y Política & 9,38 & 28,13 & 62,50 & 100,00 \\
\hline Análisis Financiero en Marketing & 60,00 & 10,00 & 30,00 & 100,00 \\
\hline Contabilidad Costes & 0,00 & 13,04 & 86,96 & 100,00 \\
\hline Análisis Estados Contables & 10,53 & 10,53 & 78,95 & 100,00 \\
\hline Todas las asignaturas & 10,53 & 15,79 & 73,68 & 100,00 \\
\hline
\end{tabular}

Fuente: elaboración propia

Tabla 15. Valores éticos

\begin{tabular}{|c|c|c|c|c|}
\hline $\begin{array}{c}\text { ¿Considera que con esta actividad ha desarrollado } \\
\text { Ias competencias relacionadas con los valores } \\
\text { éticos? }\end{array}$ & $\begin{array}{c}\text { Desacuerdo } \\
\%\end{array}$ & $\begin{array}{c}\text { Indiferente } \\
\%\end{array}$ & $\begin{array}{c}\text { Acuerdo } \\
\%\end{array}$ & $\begin{array}{c}\text { Total } \\
\%\end{array}$ \\
\hline Eficacia y Desarrollo Profesional & 0,00 & 20,00 & 80,00 & 100,00 \\
\hline Geografía Humana, Económica y Política & 3,13 & 15,63 & 81,25 & 100,00 \\
\hline Análisis Financiero en Marketing & 0,00 & 8,33 & 91,67 & 100,00 \\
\hline Contabilidad Costes & 4,35 & 21,74 & 73,91 & 100,00 \\
\hline Análisis Estados Contables & 0,00 & 17,39 & 82,61 & 100,00 \\
\hline Todas las asignaturas & 1,67 & 17,50 & 80,83 & 100,00 \\
\hline
\end{tabular}

Fuente: elaboración propia

Tabla 16. Comunicación

\begin{tabular}{|c|c|c|c|c|}
\hline $\begin{array}{c}\text { ¿Considera que con esta actividad ha desarrollado } \\
\text { la competencia de comunicación? }\end{array}$ & $\begin{array}{c}\text { Desacuerdo } \\
\%\end{array}$ & $\begin{array}{c}\text { Indiferente } \\
\%\end{array}$ & $\begin{array}{c}\text { Acuerdo } \\
\%\end{array}$ & $\begin{array}{c}\text { Total } \\
\%\end{array}$ \\
\hline Eficacia y Desarrollo Profesional & 0,00 & 13,79 & 86,21 & 100,00 \\
\hline Geografía Humana, Económica y Política & 3,13 & 31,25 & 65,63 & 100,00 \\
\hline Análisis Financiero en Marketing & 0,00 & 0,00 & 100,00 & 100,00 \\
\hline Contabilidad Costes & 0,00 & 21,74 & 78,26 & 100,00 \\
\hline Análisis Estados Contables & 13,04 & 17,39 & 69,57 & 100,00 \\
\hline Todas las asignaturas & 3,36 & 19,33 & 77,31 & 100,00 \\
\hline
\end{tabular}

Fuente: elaboración propia

\subsection{Satisfacción con la actividad}

El último grupo de preguntas estaba referido tanto al nivel de satisfacción global con la actividad desarrollada (tabla 17) como a si el alumno recomendaría que se continuara realizando la misma en el futuro (tabla 18). En ambos casos, un 85,83\% de los alumnos han respondido afirmativamente lo que muestra un alto grado de valoración de la actividad a nivel global. 
Tabla 17. Satisfacción global con la actividad

\begin{tabular}{|c|c|c|c|c|}
\hline $\begin{array}{c}\text { Nivel de satisfacción global con la actividad } \\
\text { desarrollada }\end{array}$ & $\begin{array}{c}\text { Desacuerdo } \\
\%\end{array}$ & $\begin{array}{c}\text { Indiferente } \\
\%\end{array}$ & $\begin{array}{c}\text { Acuerdo } \\
\%\end{array}$ & $\begin{array}{c}\text { Total } \\
\%\end{array}$ \\
\hline Eficacia y Desarrollo Profesional & 3,33 & 13,33 & 83,33 & 100,00 \\
\hline Geografía Humana, Económica y Política & 3,13 & 12,50 & 84,38 & 100,00 \\
\hline Análisis Financiero en Marketing & 0,00 & 8,33 & 91,67 & 100,00 \\
\hline Contabilidad Costes & 0,00 & 8,70 & 91,30 & 100,00 \\
\hline Análisis Estados Contables & 0,00 & 17,39 & 82,61 & 100,00 \\
\hline Todas las asignaturas & 1,67 & 12,50 & 85,83 & 100,00 \\
\hline
\end{tabular}

Fuente: elaboración propia

Tabla 18. Continuidad futura de la actividad

\begin{tabular}{|c|c|c|c|c|}
\hline $\begin{array}{c}\text { ¿Recomendaría que se siguiera realizando esta } \\
\text { actividad en el futuro? }\end{array}$ & $\begin{array}{c}\text { Desacuerdo } \\
\%\end{array}$ & $\begin{array}{c}\text { Indiferente } \\
\%\end{array}$ & $\begin{array}{c}\text { Acuerdo } \\
\%\end{array}$ & $\begin{array}{c}\text { Total } \\
\%\end{array}$ \\
\hline Eficacia y Desarrollo Profesional & 3,33 & 10,00 & 86,67 & 100,00 \\
\hline Geografía Humana, Económica y Política & 3,13 & 9,38 & 87,50 & 100,00 \\
\hline Análisis Financiero en Marketing & 0,00 & 8,33 & 91,67 & 100,00 \\
\hline Contabilidad Costes & 0,00 & 8,70 & 91,30 & 100,00 \\
\hline Análisis Estados Contables & 4,35 & 21,74 & 73,91 & 100,00 \\
\hline Todas las asignaturas & 2,50 & 11,67 & 85,83 & 100,00 \\
\hline
\end{tabular}

Fuente: elaboración propia

\section{Conclusiones}

La investigación desarrollada tiene por objetivo incorporar en programas educativos universitarios la temática de la inclusión educativa y la sostenibilidad desde la óptica de los ODS. El planteamiento del trabajo se ha fundamentado en el desarrollo de competencias clave para la sostenibilidad, implementadas con un criterio transversal, en diferentes áreas de conocimiento y materias, de forma que el mensaje transmitido por los ODS quedara incluido en los programas de las asignaturas.

Los alumnos han realizado trabajos que relacionaban la materia de estudio con la sostenibilidad y los ODS, de forma que se ha conseguido una visión práctica y a la vez global de los objetivos y del contenido de la Agenda 2030. En todas las actividades realizadas, se ha utilizado la metodología flipped classroom o aula invertida que permite al alumno enfrentarse a la materia estudiada de forma reflexiva y crítica.

Los resultados de aprendizaje se han calificado mediante una rúbrica, instrumento considerado idóneo para la evaluación de competencias. La rúbrica se ha estructurado en tres apartados, en función de los objetivos de aprendizaje definidos, que a su vez han sido desarrollados a través de competencias específicas. La secuencia ha sido:

Objetivo Principal $>$ Objetivos Específicos $>$ Objetivos de aprendizaje $>$ Competencias $>$ Indicadores $>$ Nivel de Dominio. 
De esta forma se ha conseguido una evaluación de las competencias adquiridas, de forma estructurada, transparente y acorde con los objetivos de aprendizaje planteados.

La evaluación de los alumnos en las actividades realizadas se considera muy alentadora, puesto que la media de todas ellas se encuentra en el rango entre "satisfactorio" y "excelente". Ello implica que los objetivos de aprendizaje establecidos se alcanzan sobradamente en todas las materias, lo cual es importante, teniendo en cuenta que, según muestra el cuestionario cumplimentado por los alumnos, solo el 36,7\% de los alumnos conocía los ODS.

En general, los estudiantes han mostrado un alto grado de satisfacción con la actividad y además recomiendan continuar realizándola en el futuro. Asimismo, los alumnos reconocen que estas actividades no solo han incrementado su conciencia ética y social, sino que también consideran que han influido positivamente en sus perspectivas y futuro profesional. En cuanto al desarrollo de competencias, los estudiantes destacan en su valoración el aprendizaje activo y los valores éticos, seguidos de la comunicación y trabajo en equipo.

En definitiva, se considera que esta investigación ha conseguido sus objetivos en la medida que ha mostrado un método eficaz y novedoso para acercar los egresados a la sostenibilidad y los ODS, de forma reflexiva y crítica, ya que ha sido evaluada muy positivamente por los mismos y ha conseguido alcanzar los objetivos de aprendizaje y desarrollo de competencias planteados. A todas estas consideraciones hay que añadir que estos resultados son perfectamente transferibles, es decir, pueden llevarse a cabo en otra universidad o entorno docente, lo que es fundamental a la hora de medir la relevancia de la investigación educativa.

La cuestión que merece mención respecto de las limitaciones a la investigación es la relativa a la participación voluntaria de los alumnos en las encuestas, dado que existe una diferencia entre el número de alumnos que participaron en las diferentes actividades de los ODS en el aula, 166 alumnos en total, y el número de aquellos que contestaron la encuesta, 120. Es decir, una participación del $72,28 \%$ en las encuestas.

Si bien, se puede esperar que los resultados obtenidos se generalicen en instituciones de enseñanza superior, se proponen, en consecuencia, futuras líneas de investigación que aplicando una metodología análoga con datos obtenidos de otras instituciones de enseñanza superior sirvan para comparar sus resultados con los obtenidos en la presente investigación.

Por último, se ha de resaltar tanto el apoyo recibido por la Universidad Europea para llevar a cabo el proyecto de investigación-acción, como la oportunidad brindada a los investigadores de presentar los resultados en la mesa redonda "Los ODS en la Universidad Europea de Madrid", enmarcada dentro de la jornada "ODS Day", celebrada el día 9 de mayo de 2019.

\section{Referencias}

Aguilera, C., Manzano, A., Martínez, I., Lozano, M. C., y Casiano, C. (2017). El modelo flipped classroom. International Journal of Developmental and Educational Psychology, 4(1), 261 266. DOI: https://doi.org/10.17060/ijodaep.2017.n1.v4.1055

Bergmann, J., \& Sams, A. (2012). Flip your classroom: Reach every student in every class every day. United States of America: International Society for Technology in Education.

Buján, K., Rekalde, I., y Aramedi, P. (2011). La evaluación de competencias en la educación superior: Las rúbricas como instrumento de evaluación. Alcalá de Guadaira, Sevilla: Paréntesis Editorial. 
Crimmins, M. \& Midkiff, B. (2017). High Structure Active Learning Pedagogy for the Teaching of Organic Chemistry. Assessing the Impact on Academic Outcomes. Journal of chemical education, 94(4), 429-438. DOI: https://doi.org/10.1021/acs.jchemed.6b00663

Hu, R., Gao, H., Ye, Y., Ni, Z., Jiang, N., \& Jiang, X. (2018). Effectiveness of flipped classrooms in Chinese baccalaureate nursing education: A meta-analysis of randomized controlled trials. International Journal of Nursing Studies, 79, 94-103. DOI: http://doi.org/10.1016/j. ijnurstu.2017.11.012

Jordan, C., Pérez, M. J., y Sanabria, E. (2014). Investigación del impacto en un aula de matemática al utilizar "flip education". Revista "Pensamiento Matemático", 4(2), 9-22.

Martínez, M. (2000). La investigación-acción en el aula. Agenda académica, 7(1), 27.

Mattis, K. V. (2015). Flipped classroom versus traditional textbook instruction: Assessing accuracy and mental effort at different levels of mathematical complexity. Technology, Knowledge and Learning, 20, 231-248. DOI: https://doi.org/10.1007/s10758-014-9238-0

Matzumura, J. P., Gutiérrez, H., Zamudio, L. A., y Zavala, J. C. (2018). Aprendizaje invertido para la mejora y logro de metas de aprendizaje en el curso de metodología de la investigación en estudiantes de universidad. Revista Electrónica Educare, 22(3), 1-21. DOI: http://dx.doi.org/10.15359/ree.22-3.9

Programa de las Naciones Unidas para el Desarrollo. PNUD. (2019). Objetivos de Desarrollo Sostenible. Recuperado de https://www.undp.org/content/undp/es/home/sustainable-development-goals.html. Último acceso 1 de junio de 2020.

Sola, T., Aznar, I., Romero, R., y Rodríguez-García, A. (2019). Eficacia del Método Flipped Classroom en la Universidad: Meta-Análisis de la Producción Científica de Impacto. REICE. Revista Iberoamericana sobre Calidad, Eficacia y Cambio en Educación, 17(1), 25-38. DOI: https://doi.org/10.15366/reice2019.17.1.002

Terrón, M. J., Velasco, P., y García, M. J. (2012) Guía para el diseño de recursos docentes que fomenten el desarrollo y evaluación de las competencias transversales en educación. España: Editorial Fundación Vértice Emprende.

Tourón, J., Santiago, R., y Díez, A. (2014). The flipped classroom. Cómo invertir la escuela en un espacio de aprendizaje. Barcelona: Grupo Océano.

United Nations Educational, Scientific and Cultural Organization. UNESCO. (2015). Global citizenship education: topics and learning objectives. Recuperado de https://unesdoc.unesco.org/ark:/48223/pf0000232993. Último acceso 12 de junio de 2020.

United Nations Educational, Scientific and Cultural Organization. UNESCO. (2017). Education for Sustainable Development Goals: Learning Objectives. Recuperado de: https:// www.sdg4education2030.org/education-sustainable-development-goals-learning-objectives-unesco-2017. Último acceso 10 de junio e 2020.

\section{nORMATIVA}

Comisión Europea. (2016). Comunicación de la Comisión al Parlamento Europeo, al Consejo, al Comité Económico y Social Europeo y al Comité de las Regiones, de 22 de noviembre de 2016, "Próximas etapas para un futuro europeo sostenible - Acción europea para la sostenibilidad". COM (2016) 740 final. Estrasburgo. 
Comunicación de la Comisión al Parlamento Europeo, al Consejo y al Comité Económico y Social Europeo, de 22 de marzo de 2006. "Poner en práctica la asociación para el crecimiento y el empleo: hacer de Europa un polo de excelencia de la responsabilidad social de las empresas". COM (2006) final.

Naciones Unidas. (2015). "Transformando nuestro mundo: la Agenda 2030 para el desarrollo sostenible”, resolución adoptada el 25 de septiembre de 2015 por la Asamblea General de las Naciones Unidas (AGNU A/RES/70/1). 